\title{
Theoretical Line Loss Calculation System of Low-voltage Distribution Network Based On Three-phrase Dynamic Unbalance
}

\author{
Zhang ShiHong ${ }^{1, a}$, Yu JlanGMing ${ }^{2, b}$ \\ ${ }^{1}$ Department of Electrical Engineering, Xi'an University of Technology, Xi'an, Shaanxi Province, \\ 710048, China \\ ${ }^{2}$ Department of Electrical Engineering, Xi'an University of Technology, Xi'an, Shaanxi Province, \\ 710048, China \\ aemail: 24190150@qq.com, bemail: jianmingyu@xaut.edu.cn
}

Keywords: low-voltage network; theoretical loss calculation; three-phase unbalanced degree.

\begin{abstract}
In considering the effect of three-phase unbalance degree upon the low voltage line loss, the dynamic three-phase unbalance coefficient is adopted to improve the computation method for low-voltage line loss based on equal electric resistance method in this paper.With an aim at the most of low-voltage network without collecting the representative daily current data, the three-phase unbalance degree acquisition device is designed in this paper, which adopts a single chip as its master controller. It is connected to the output terminal of transformer to collect and save the real-time three-phase currents for dynamic unbalance degree calculation. A real low-voltage distribution network is used to test the improved computing method, and to compare the results obtained with the traditional computing method.The results from computing examples indicate that this computing system is feasible and has high potential values in engineering applications, with the calculated results nearing to real value of line loss.
\end{abstract}

\section{Introduction}

The operation optimization and loss-reduction measures of the low-voltage distribution network have been an great concern issue to the power supply enterprise, which is of great significance to the reasonable investment and improvement of economic benefits of the power supply enterprises [1]. The most commonly used line loss calculation methods of the low-voltage distribution network mainly include the Rms current method, the average current method, the maximum current method, the equivalent resistance method, and the hours of the maximum load loss method etc [2-3]. However, the imbalance running case of the low-voltage distribution network is not considered in above methods, which makes the theoretical line loss calculation of the low-voltage distribution network not reflect the true situation of the grid operation. But the hourly three-phase current of the representative day must be known to get the three-phase instantaneous imbalance. That is to say, the load tester should be installed at each distribution transformer of the station area, which is not easy to achieve in practice. According to this situation, a line loss calculation model considering dynamic three-phase imbalance is presented in this paper. What's more, the three-phase unbalance acquisition device is introduced aiming at the impossibility to get three-phase current, so that the calculated results can be well close to the actual value.

\section{Relation Between Line Losses and Unbalance}

When three-phase load is unbalanced, the load current of each phase is not equal, which induces current imbalance between phase. These unbalance currents not only cause loss in phase line but also cause loss in the neutral line, increasing the total line loss [4-5].

Define the unbalance of load current as

$$
\beta_{\varphi}=\frac{I_{\varphi}-I_{\mathrm{av}}}{I_{\mathrm{av}}} \times 100 \%, \quad \beta_{\mathrm{a}}=\frac{I_{\mathrm{a}}-I_{\mathrm{av}}}{I_{\mathrm{av}}} \times 100 \%, \quad \beta_{\mathrm{b}}=\frac{I_{\mathrm{b}}-I_{\mathrm{av}}}{I_{\mathrm{av}}} \times 100 \%, \quad \beta_{\mathrm{c}}=\frac{I_{\mathrm{c}}-I_{\mathrm{av}}}{I_{\mathrm{av}}} \times 100 \%
$$


Where, $I_{\varphi}$ is phase current, $I_{\mathrm{a}} 、 I_{\mathrm{b}} 、 I_{\mathrm{c}}$ is three-phase load current, $I_{\mathrm{av}}$ is average load current, $I_{\mathrm{av}}$ $=\left(I_{\mathrm{a}}+I_{\mathrm{b}}+I_{\mathrm{c}}\right) / 3$. Obviously, $I_{\varphi}=\left(1+\beta_{\varphi}\right) / I_{\mathrm{av}}$. Because $I_{\varphi}$ ranges from 0 to $I_{\mathrm{av}}$, so the range of $\beta_{\varphi}$ is $-1 \sim 2$, and

$$
\beta_{\mathrm{a}}+\beta_{\mathrm{b}}+\beta_{\mathrm{c}}=0
$$

Assuming that the phase-line resistance and neutral-line resistance of the three-phase four-line system are all R, and the power factor of each phase is same, so when three phases are imbalance, the power loss of the phase-line is

$$
\Delta P_{\varphi}=\left[\left(1+\beta_{\mathrm{a}}\right)^{2}+\left(1+\beta_{\mathrm{b}}\right)^{2}+\left(1+\beta_{\mathrm{c}}\right)^{2}\right] I_{\mathrm{av}}^{2} R=\left(3+{\beta_{\mathrm{a}}}^{2}+{\beta_{\mathrm{b}}}^{2}+{\beta_{\mathrm{C}}}^{2}\right) I_{\mathrm{av}}^{2} R
$$

The current of the neutral-line is

$$
\dot{I}_{\mathrm{N}}=\left(1+\beta_{\mathrm{a}}\right) I_{\mathrm{av}}+\left(1+\beta_{\mathrm{b}}\right)\left(-\frac{1}{2}-\mathrm{j} \frac{\sqrt{3}}{2}\right) I_{\mathrm{av}}+\left(1+\beta_{\mathrm{c}}\right)\left(-\frac{1}{2}+\mathrm{j} \frac{\sqrt{3}}{2}\right) I_{\mathrm{av}}
$$

So

$$
I_{\mathrm{N}}^{2}=\left(\beta_{\mathrm{a}}^{2}+\beta_{\mathrm{b}}^{2}+\beta_{\mathrm{c}}^{2}-\beta_{\mathrm{a}} \beta_{\mathrm{b}}-\beta_{\mathrm{b}} \beta_{\mathrm{c}}-\beta_{\mathrm{c}} \beta_{\mathrm{a}}\right) I_{\mathrm{av}}^{2}
$$

The power loss of neutral-line is

$$
\Delta P_{\mathrm{N}}=I_{\mathrm{N}}^{2} R
$$

So the total loss is

$$
\Delta P_{\text {unb }}=\Delta P_{\varphi}+\Delta P_{\mathrm{N}}=\left[3+2\left(\beta_{\mathrm{a}}^{2}+\beta_{\mathrm{b}}^{2}+\beta_{\mathrm{c}}^{2}\right)-\left(\beta_{\mathrm{a}} \beta_{\mathrm{b}}+\beta_{\mathrm{b}} \beta_{\mathrm{c}}+\beta_{\mathrm{c}} \beta_{\mathrm{a}}\right)\right] I_{\mathrm{av}}^{2} R
$$

Where, $3+2\left(\beta_{\mathrm{a}}^{2}+\beta_{\mathrm{b}}^{2}+\beta_{\mathrm{c}}^{2}\right)-\left(\beta_{\mathrm{a}} \beta_{\mathrm{b}}+\beta_{\mathrm{b}} \beta_{\mathrm{c}}+\beta_{\mathrm{c}} \beta_{\mathrm{a}}\right)$ is the unbalance factor.

\section{Improvement of the Low-Voltage Line Loss Calculation Algorithm}

\section{Three-phase dynamic unbalance determined based on acquisition phase current}

Three-phase dynamic unbalance is general a fixed value, which be calculated based on the definition of three-phase unbalance. That is to say, the line loss calculation of all moment adopts the unbalance value that is calculated in a typical moment, which is clearly inappropriate. A dynamic unbalance value should be adopted to calculate the low-voltage line loss precisely.

If three-phase currents $I_{\mathrm{a} \cdot i}, I_{\mathrm{b} \cdot i}, I_{\mathrm{c} \cdot i}$ of the low-voltage stage area can be acquired by first section of the stage area within $24 \mathrm{~h}$ and separated by $\mathrm{t}(\mathrm{h})$, so three-phase current curves can be got. Here believe that load does not change during $t$, so the shorter $t$ is, the more the number of samples is. As a result, the higher accuracy of the final calculation can be got

Then three-phase average current $I_{\mathrm{av} \cdot i}$ curves can be got by the function $I_{\mathrm{av}}=\left(I_{\mathrm{a}}+I_{\mathrm{b}}+I_{\mathrm{c}}\right) / 3$. And based on equation (12), three-phase unbalance $\beta_{\mathrm{a} \cdot i}, \beta_{\mathrm{b} \cdot i}, \beta_{\mathrm{c} \cdot i}$ curves can be calculated.

$$
\beta_{\mathrm{a} \cdot i}=\frac{I_{\mathrm{a} \cdot i}-I_{\mathrm{av} \cdot i}}{I_{\mathrm{av} \cdot i}} \times 100 \%, \quad \beta_{\mathrm{b} \cdot i}=\frac{I_{\mathrm{b} \cdot i}-I_{\mathrm{av} \cdot i}}{I_{\mathrm{av} \cdot i}} \times 100 \%, \quad \beta_{\mathrm{c} \cdot i}=\frac{I_{\mathrm{c} \cdot i}-I_{\mathrm{av} \cdot i}}{I_{\mathrm{av} \cdot i}} \times 100 \%
$$

Where, $i=0-t$.

So three-phase dynamic unbalance can be given as

$$
K_{1 \cdot i}=3+2\left(\beta_{\mathrm{a} \cdot i}^{2}+\beta_{\mathrm{b} \cdot i}^{2}+\beta_{\mathrm{c} \cdot \mathrm{i}}^{2}\right)-\beta_{\mathrm{a} \cdot \mathrm{i}} \beta_{\mathrm{b} \cdot i}-\beta_{\mathrm{a} \cdot i} \beta_{\mathrm{c} \cdot \mathrm{i}}-\beta_{\mathrm{b} \cdot \mathrm{i}} \beta_{\mathrm{c} \cdot i}
$$

Thus three-phase dynamic unbalance is no longer a fixed value, and will change with time.

\section{Determine of the average current of dynamic load}

The average daily power supply can be derived by the user's total electricity consumption of the month $A_{\text {month }}$

$$
A_{\text {day }}=A_{\text {month }} / N
$$

Where, $N$ is the number of the day.

Derive three-phase average current $I_{\mathrm{av} \cdot i}$ from $I_{\mathrm{av}}=\left(I_{\mathrm{a}}+I_{\mathrm{b}}+I_{\mathrm{c}}\right) / 3$, then decompose the average daily power supply according to the current squared curve, and the power supply for each $\mathrm{t}(\mathrm{h})$ time period can be got 


$$
A_{\mathrm{t} \cdot i}=\frac{A_{\mathrm{day}}}{\sum_{i=1}^{n} I_{\mathrm{av} \cdot i}} \times I_{\mathrm{av} \cdot i}
$$

Where, $A_{\mathrm{t} \cdot \mathrm{i}}$ is power supply for each $\mathrm{t}(\mathrm{h})$ time period; $I_{\mathrm{av} \cdot \mathrm{i}}$ is three-phase average current in the $\mathrm{t}$ section.

At last, the average load current of line first section in the t period is given

$$
I_{\mathrm{pj} i}=\frac{A_{\mathrm{t} \cdot \mathrm{i}}}{\sqrt{3} U_{\mathrm{av}} \cos \varphi}
$$

\section{Determine of the calculation model based on improved algorithm}

Based on previous three-phase dynamic unbalance and dynamic load average current, the improved model of the stage area line loss is given as

$$
\Delta A_{1}=K_{2}^{2} \sum_{i=1}^{n} K_{1 \cdot i} I_{\mathrm{pj} \cdot i}^{2} R_{\mathrm{eq}} \frac{T}{24} \times 10^{-3}(\mathrm{kWh})
$$

Where, $K_{1 \cdot i}$ is three-phase dynamic balance, $K_{2}$ is load curves factor of line headend; $I_{\mathrm{pj} \cdot i}$ is the average load current of of line headend at section; $n$ is the transcribing number of load current on representative day; $T$ is the electricity assumption time (h).

This calculation model makes three-phase unbalance of each separated $t(h)$ multiplied by the load average current of each separated $t(h)$, and then added to the system power loss, which reflects the time-varying of the system, thereby improve the calculation accuracy.

\section{Line Loss Calculation Procedure of Low-voltage Stage Area}

The line loss of low-voltage stage area refers to the power loss of the distribution equipment between total meter (generally set at low-voltage side of the distribution transformer) and the user's meter. Calculation procedure show in Figure 2.

(1) Calculate the equivalent resistance of low-voltage station area

Divide the entire low-voltage station area into some calculation segments from the first end to end. Principles of the segment division: If the transporting load, the choice of line number, the length of the segment are the same, they are same calculation segment. So $R_{\text {eq }}$ is as follows:

$$
\begin{gathered}
R_{\mathrm{eq}}=\frac{\sum_{j=1}^{n} N_{j} A_{j \cdot \Sigma}^{2} R_{j}}{N\left(\sum_{i=1}^{m} A_{i}\right)^{2}} \\
R_{j}=r_{\mathrm{o} \cdot j} \times L_{j}
\end{gathered}
$$

Where, $A_{i}$ is energy meter electricity consumption of each load points in the low-voltage distribution network $(\mathrm{kWh}) ; A_{j \cdot \Sigma}$ is energy meter electricity consumption of some segment in the low-voltage distribution network $(\mathrm{kWh}) ; R_{j}$ is the resistance of a calculation-segment line $(\Omega) ; r_{0 \cdot j}$ is the unit length resistance of each calculation-segment line $(\Omega / \mathrm{km}) ; L_{j}$ is the length of each calculation-segment line $(\mathrm{km}) ; N$ is the structure constant of the low-voltage terminal of the distribution transformer, which equals to 2 in single-phase two-line system, 3 in three-phase three-line system and 3.5 in three-phase four-line system.

(2) Calculate line loss according to equation (17).

(3) Calculate the fixed energy loss.

The energy loss includes loss of low-voltage capacitance, current transformer, voltage transformer, three-phase ammeter and single-phase ammeter.

The total loss of the station area

$$
\Delta A_{\Sigma}=\Delta A_{1}+\Delta A_{\mathrm{b}}
$$




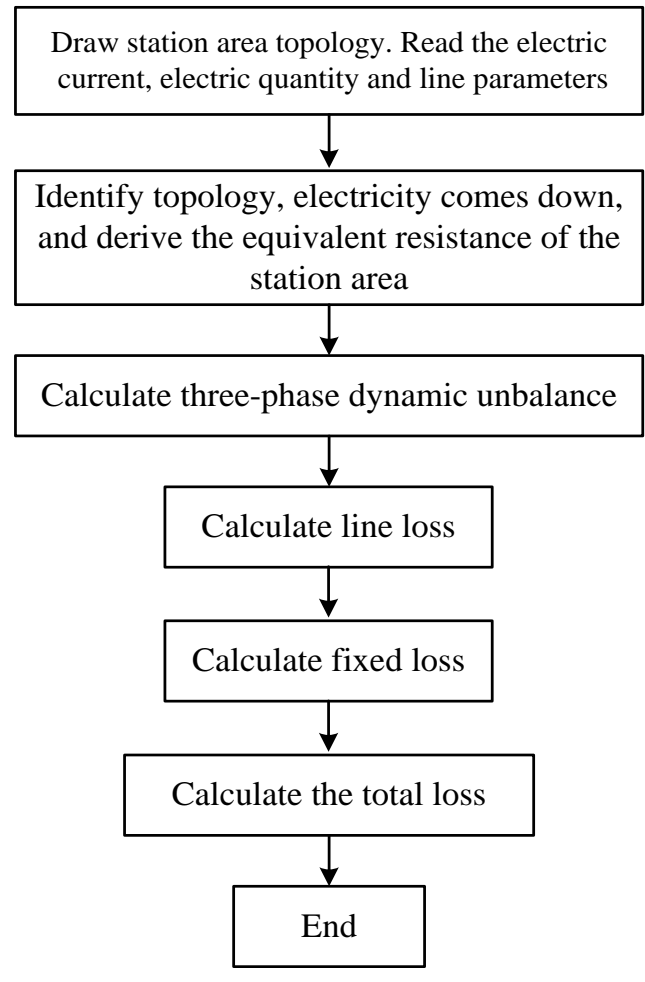

Figure 1. Calculation steps.

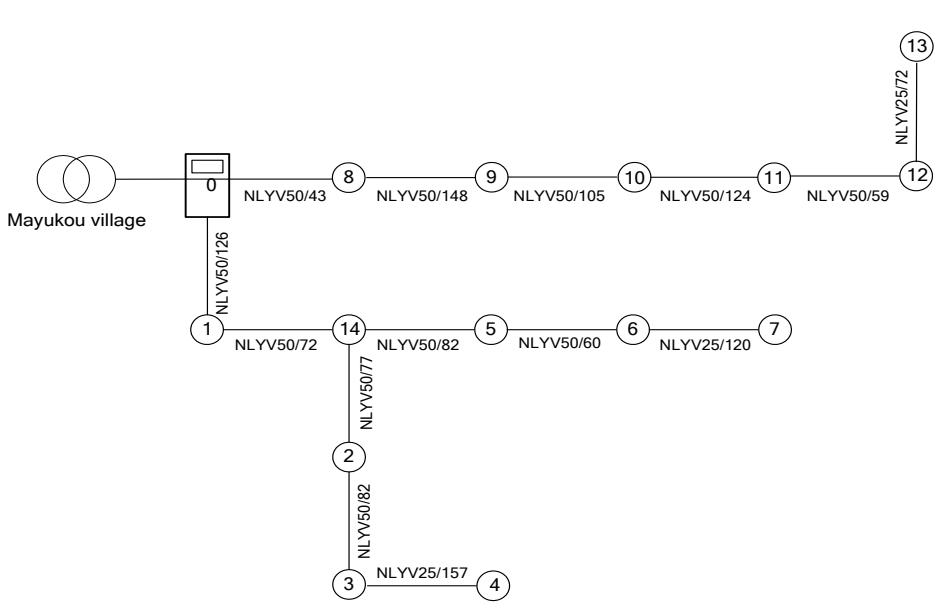

Figure 2. Low-voltag distribution lines network.

\section{Case Analysis}

In this paper, an actual running stage area of Tongguan County Electricity Authority, located in Weinan City, Shaanxi Province is regarded as the study case, the actual network structure shown in Figure 2. The conventional equivalent resistance method and the improved equivalent resistance method are adopted to calculate the theoretical line loss respectively.

The total electricity consumption of this station area in August 2007 is $16950 \mathrm{kWh}$, the power factor is 0.85 , the characteristic coefficient of load curve is1.25, the running time is $744 \mathrm{~h}$, and the average phase voltage is $0.22 \mathrm{kV}$. The line parameters show in Fig. 3, and other relevant parameters show in Table 1,2,3.

Table 1. Parameter of load

Table 2. Parameter of fixed wastage

\begin{tabular}{cc||cc}
\hline $\begin{array}{c}\text { Meter } \\
\text { NO. }\end{array}$ & $\begin{array}{c}\text { Electricity } \\
(\mathrm{kWh})\end{array}$ & phase & $\begin{array}{c}\text { Electricity } \\
(\mathrm{kWh})\end{array}$ \\
\hline 1 & 537 & 8 & 335 \\
2 & 260 & 9 & 470 \\
3 & 355 & 10 & 567 \\
4 & 3145 & 11 & 391 \\
5 & 5795 & 12 & 2578 \\
6 & 296 & 13 & 399 \\
7 & 206 & 14 & 0 \\
\hline
\end{tabular}

\begin{tabular}{ccc}
\hline Type & loss $\left(\mathrm{kWh} \cdot \mathrm{month}^{-1}\right)$ & NO. \\
\hline LV capacitance & 0.5 & 2 \\
Current transformer & 0.5 & 2 \\
Voltage transformer & 0.5 & 2 \\
Single-phase meter & 1 & 167 \\
Three-phase meter & 2 & 38 \\
\hline
\end{tabular}

From the data in Table 5, the calculation results of the improved algorithm are large than the traditional algorithm, which is mainly caused by the consideration of the influence of three-phase unbalance on the line loss. Then it can clearly see from Table 4 that three-phase unbalance coefficient $\mathrm{K} 1$ is changing with time. The influence on line losses is different at each time segment, the largest unbalance factor is 5.95, the line loss is about 2 times in this period than the load run in balance. Therefore, the proposed algorithm is that multiply three-phase unbalance of each period 
Table 3. Parameter of Current

\begin{tabular}{cccc||cccc}
\hline$t$ & $\mathrm{I}_{\mathrm{a}} / \mathrm{A}$ & $\mathrm{I}_{\mathrm{b}} / \mathrm{A}$ & $\mathrm{I}_{\mathrm{c}} / \mathrm{A}$ & $t$ & $\mathrm{I}_{\mathrm{a}} / \mathrm{A}$ & $\mathrm{I}_{\mathrm{b}} / \mathrm{A}$ & $\mathrm{I}_{\mathrm{C}} / \mathrm{A}$ \\
\hline 0 & 14 & 23.2 & 14.8 & 12 & 30.4 & 90.1 & 70.8 \\
1 & 9.56 & 25.8 & 13.5 & 13 & 31.8 & 69.1 & 90.8 \\
2 & 8.14 & 28.2 & 8.52 & 14 & 32.7 & 84 & 81.6 \\
3 & 9.28 & 11.2 & 7.5 & 15 & 30 & 44.4 & 45.3 \\
4 & 9.7 & 12.8 & 7.8 & 16 & 20.5 & 52.5 & 45.3 \\
5 & 9.79 & 12.1 & 2.4 & 17 & 26.9 & 71.1 & 68.7 \\
6 & 9.68 & 8.8 & 9.5 & 18 & 39.3 & 105 & 109 \\
7 & 20.2 & 20 & 21.7 & 19 & 30.6 & 89.6 & 90.2 \\
8 & 21.8 & 61.5 & 56.7 & 20 & 38.9 & 72.9 & 70.8 \\
9 & 22.4 & 63.6 & 57.6 & 21 & 28.7 & 80.6 & 65.7 \\
10 & 22.6 & 68.1 & 65.7 & 22 & 25.2 & 68.9 & 50.7 \\
11 & 28.7 & 78 & 72.9 & 23 & 21.3 & 50.8 & 46.7 \\
\hline
\end{tabular}

Note: $t$ is acquisition session.

Table 5. The calculation result

\begin{tabular}{cccc}
\hline Calculation model & $\begin{array}{c}\text { Theoretical } \\
\text { total loss(kWh) }\end{array}$ & $\begin{array}{c}\text { Theoretical } \\
\text { line-loss rate(\%) }\end{array}$ & $\begin{array}{c}\text { Statistics line-loss } \\
\text { rate(\%) }\end{array}$ \\
\hline $\begin{array}{c}\text { Equivalent resistance } \\
\text { method }\end{array}$ & 1071.66 & $6.32 \%$ & $9.53 \%$ \\
$\begin{array}{c}\text { Improved equivalent } \\
\text { resistance method }\end{array}$ & 1410.24 & $8.32 \%$ & $9.53 \%$ \\
\hline
\end{tabular}

separated by t (h) by the average load current of each period separated by $\mathrm{t}(\mathrm{h})$, and then added to the system loss, which reflects the time-varying characteristics, to make the results more close to the true value of the line loss.

\section{Conclusion}

In this paper, the improved ideological equivalent method to calculate line loss is proposed based on the improvement of the ideological equivalent method and the consideration of the influence of the three-phase unbalanced on line loss. The improved ideological equivalent method makes the unbalance value more close to the realistic values at different period, so this method improves the accuracy of line loss calculation. Then three-phase unbalance acquisition device with a strong practical value is designed. The system has been programmed and put into use with good effect.

\section{References}

[1] BO Yonghong. Influence on Technical Loss by Three-phase Load Unbalanced in Distribution Networks [J]. Popular Utilization of Electricity,2004,(12):30-31.

[2] Cai Shujin. Influence on Circuit Loss by Three-phase load Unbalanced [J]. Rural Electrification,2013,4: 23-24.

[3] Dong Jizheng, Cheng Hongyun, He Yigang. Method for Calculating Transformer District Line Loss Based on Load Classification [J]. Modern Electric Power,2006,23(1):44-48.

[4] Gao Junqin. The circuit loss rate analysis of three-phase unbalanced circuit [J]. Journal of Electric Power,2009,16(2): 91-93.

[5] Hu Hongyan, Wen Buying. Lower-voltage distribution network circuit theoretical loss[J]. Rural Electrification, 2012(11):47-48. 